\title{
Nématodes pulmonaires des antilopes d'Afrique centrale
}

\author{
par M. GRABER $\left(^{*}\right)$, J. EUZEBY $\left({ }^{*}\right)$, P. M. TRONCY $(* *)$ et J. THAL $\left(^{* *}\right)$
}

\begin{abstract}
RESUME
Une pneumonie vermineuse due à la présence de Pneumostrongylus cornigerus (Ortlepp, 1962) dans les alvéoles pulmonaires du Damalisque, de l'Hippotrague et du Bubale rouge est signalée dans l'est de la R.C.A. Ce sont les premiers cas observés, au nord de l'équateur, chez des ruminants sauvages. L'Hippotrague et le Bubale rouge représentent des hôtes nouveaux. Parasite et lésions sont brièvement redécrits.
\end{abstract}

Actuellement, on dénombre en Afrique neuf espèces de Nématodes, parasites des voies respiratoires des ruminants sauvages.

Deux d'entre elles font partie des Dictyocaulidae :

- Dictyocaulus viviparus BLOCH, 1782 RAILLIET et HENRY, 1907 qui a été découvert dans les bronches de l'Elan du Cap (Taurotragus oryx), du Gnou (Chonnochaetes taurinus), du Bubale (Alcelaphus cokei) et du Damalisque (Damaliscus korrigum) au zoo Groote Schuur du Cap (11) et au parc de Serengeti en Tanzanie $(14,2)$.

- Dictyocaulus magnus MóNNIG, 1932 DOUGHERTY, 1946 du Damalisque et du Springbuck (Antidorcas marsupialis). Il a été recueilli en divers points d'Afrique du Sud (Etat d'Orange; Lombart nature reserve au Transvaal) par MÖNNIG (7) et ORTLEPP (11) et au parc national de Washington (1).

Les sept autres sont des Protostrongylidae appartenant à trois genres différents: Pneumostrongylus, Protostrongylus et Müllerius.

(*) Laboratoire de Parasitologie, Ecole Nationale Vétérinaire, 2, quai Chauveau, 69337 Lyon, Cedex 1, France. Tchad.
- Sur le continent africain, l'espèce la plus anciennement connue est Pneumostrongylus calcaratus décrite par MóNNIG en 1932 (8) à partir de fragments prélevés dans les bronchioles et les alvéoles d'un Impala (Aepyceros melampus) originaire du Transvaal. Ultérieurement, le Nématode, qui est le plus commun et le moins spécifique des Protostrongylidae d'Antilopes avec lesquels il est souvent associé, a été retrouvé chez des Impala du parc Krüger (11), de la réserve Sabie-Sand game (6) et du parc Serengeti (2), ainsi que chez le Gnou, le Bubale, le Damalisque, la Gazelle de Thomson (Gazella thomsoni) et la Gazelle de Grant (Gazella granti) en Tanzanie (14).

- Millerius capillaris MULLER, 1889 CAMERON, 1927 a été signalé, dès 1936, au Kenya chez l'Impala et la Gazelle de Grant (13). Il a également été identifié chez l'Okapi (Okapia johnstoni) à partir des fèces de cet animal (5).

- Protostrongylus gazellae (16) affecte plus particulièrement les Gazelles (Centre de la Tanzanie et Serengeti).

- Protostrongylus capensis (12) n'a été vu qu'une seule fois chez les Damalisques de la réserve Bontebok au Cap. 
- Protostrongylus etoshai (12) existe dans le Sud-Ouest Africain (réserve d'Etosha) et en Tanzanie (Serengeti). Les hôtes sont le Gnou, l'Oryx, le Damalisque et le Bubale.

- Pneumostrongylus cornigerus (12) siège dans les alvéoles pulmonaires des Damalisques et des Bubales (Old Bontebok national Park, Province du Cap et Serengeti).

- Protostrongylus africanus (2) espèce de grande taille, vit dans les bronches et les bronchioles du Damalisque, du Bubale, du Gnou et, plus rarement, de l'Impala et de la Gazelle de Thomson (Tanzanie: Serengeti).

Les Protostrongylidae frappent donc les Bovidae sauvages des sous-familles des Antilopinae, des Alcelaphinae, des Oryginae et des Tragelaphinae, à l'exclusion des Bovinae, des Reduncinae, des Raphicerinae et des Cephalophinae. En outre, toutes les récoltes ont été effectuées, jusqu'à présent, au sud de l'équateur, dans des pays anglophones, ou sur des animaux de zoo provenant de ces régions.

Au cours des années 1970 et 1971, l'autopsie de quatre Hippotragues (Hippotragus equinus), d'un Damalisque (Damaliscus korrigum) et de six Bubales rouges (Alcephalus lelwel), abattus par l'un d'entre nous dans l'est de la République centrafricaine, a permis de mettre en évidence des lésions de pneumonie vermineuse due à Pneumostrongylus cornigerus.

Les taux d'infestation sont élevés : six Bubales sur 24 , soit près du quart des animaux sacrifiés; quatre Hippotragues sur 10.

C'est la première fois qu'un Nématode pulmonaire de la grande faune africaine est découvert dans l'hémisphère nord.

L'Hippotrague et le Bubale rouge représentent des hôtes nouveaux. La spécificité de Pneumostrongylus cornigerus n'est donc pas limitée aux Alcelaphinae, comme le pensent DINNIK et SACHS (2): elle s'étend également aux Oryginae ou Hippotraginae.

Aucun Protostrongylidae n'a été rencontré chez le Buffle [Bubalus (Syncerus) caffer] le Waterbuck (Kobus defassa), le Cob de Buffon (Adenota $K o b$ ), le Cervicapre de roseaux (Redunca redunca), l'Ourébi (Ourebia ourebi), le Cephalophe (Sylvicapra grimmia), l'Elan de
Derby (Taurotragus derbianus) et le Guib (Tragelaphus scriptus), soit au total 138 animaux

Trois foyers (carte) ont pu être ainsi délimités :

1. Est du Dar el Kouti, Massif des Bongos, Goula, c'est-à-dire une zone traversée par les rivières Gounda, Sakala, Kapa et Vakaga $\left(218 \mathrm{Cd}\left(^{*}\right), 218 \mathrm{Da}, 218 \mathrm{Dd}, 219 \mathrm{Bb}\right.$, $229 \mathrm{Ca}$ : deux Hippotragues, trois Bubales et un Damalisque.

2. Région du Bangoran, au Nord-Ouest de N'Délé (19 8 D b) : deux Hippotragues.

3. Rivière Voulou $(237 \mathrm{Cd})$ et Sud d'Ouandjia (23 $8 \mathrm{~A} \mathrm{~b}$ ) : trois Bubales. L'ensemble des foyers (carte) est situé entre le septième et le dixième parallèle qui semble marquer, en Afrique centrale, la limite nord de l'aire de répartition du parasite $(* *)$. Ce sont des zones où la saison des pluies dure six mois (de mai à novembre), avec des précipitations supérieures à $1.400 \mathrm{~mm}$ et plus de 90 jours de pluie. Le degré hygrométrique reste élevé. Ces conditions sont éminemment favorables au développement et à la survie des mollusques, hôtes intermédiaires des Protostrongylidae pulmonaires.

Le parasite et les lésions qu'il détermine méritent de retenir un peu plus l'attention.

\section{LE PARASITE}

Les lésions ayant été disséquées après fixation au formol, seuls des fragments de 8,8 à $12,4 \mathrm{~mm}$ ont pu être isolés dont 10 bourses caudales de mâles et 4 extrémités antérieures et postérieures de mâles et de femelles.

Les vers dont les éléments mesurables sont donnés au Tableau $\mathrm{n}^{\mathrm{o}}$ I sont filiformes, blanchâtres, et les femelles un peu plus larges que les mâles.

La tête porte une bouche, pourvue de trois petites lèvres, qui aboutit directement dans l'œsophage. Celui-ci, de forme cylindrique, est un peu plus étroit dans la région antérieure que dans la région postérieure.

(*) Coordonnées I.B.A.H.

(*⿻) Qui, jusquà maintenant, n'a pu être isolé au Tchad sur un total de 164 antilopes examinées. 


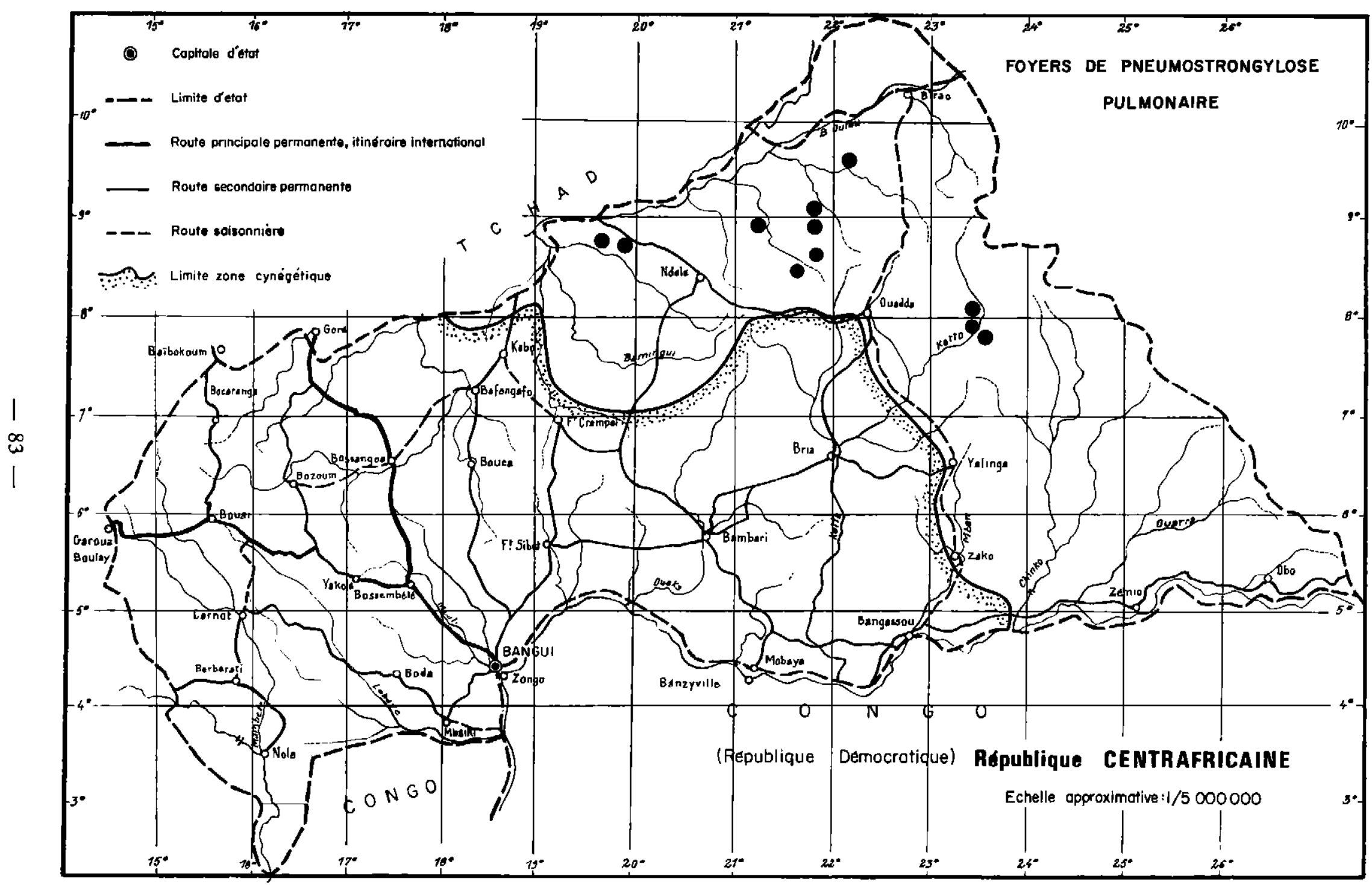


TABLEAL N* I

Mensurations (en $\mu$ ) de Pneumostrongylus comigerus

\begin{tabular}{|l|c|c|}
\hline & Mâle & Femelle \\
\cline { 2 - 3 } $\begin{array}{l}\text { Largeur du corps } \\
\text { Largeur de la tête }\end{array}$ & $\begin{array}{c}100-120 \\
\text { Oesophage } \\
\text { Longueur }\end{array}$ & $\begin{array}{c}160-200 \\
45-48\end{array}$ \\
$\begin{array}{l}\text { Largeur } \\
\text {-Portion antêrieure } \\
\text {-Portion postêrieure }\end{array}$ & 340 & $400-416$ \\
Distance anneau nerveux & 54 & 40 \\
à extrémitế antêrieure & 168 & 60 \\
Longueur des spicules \\
(moyenne) & 470 & 200 \\
-Manche & 183,6 & \\
-Lame & 286,4 & \\
Gubernaculum & & \\
-crura & $50-60$ & \\
-corpus & $75-80$ & 220 \\
Distance vulve-anus & & $100-104$ \\
Distance anus-queue & & \\
\hline
\end{tabular}

La bourse caudale du mâle (planche I, fig. I) est formée de deux lobes latéraux et d'un lobe dorsal peu visible. Les côtes ventrales sont fusionnées sur les deux tiers de leur longueur. Le système latéral (planche I, fig. I) comprend :

- Une côte antéro-latérale courte, trapue, bien individualisée.

- Les côtes médio-latérale et postéro-latérale réunies en un tronc unique qui, à son extrémité, se divise en deux branches. Il est le seul à atteindre le bord de la bourse caudale.

La côte externo-dorsale est nettement séparée des précédentes. La côte dorsale, épaisse, est de taille réduite (planche I, fig. 2).

Les spicules (planche I, fig. 1 et 3 ), égaux, brun-noirâtre, mesurent de 452 à $488 \mu$ (en moyenne $470 \mu$ ). Leur structure est double: la partie proximale (= Le manche), arrondie et granuleuse, est plus courte (rapport 2/5) que la partie distale (= La lame). Celle-ci, élargie et aplatie, est pourvue d'ailes bien développées soutenues par des épaississements cuticulaires.

Le gubernaculum (planche I, fig. 4), comme chez tous les Protostrongylidae, est un organe complexe composé :

- de deux pièces chitineuses, les crura, de couleur brune, en forme de crochets dont la pointe n'est pas recourbée. Elles portent, à leur bord interne, deux petits appendices plus clairs;
- d'une partie moyenne, le corpus, faiblement chitinisé, long de 75 à $80 \mu$;

- le capitulum, est absent.

La queue de la femelle (planche II, fig. 1) se termine en pointe. La vulve est située à $220 \mu$ en avant de l'anus et cette région est couverte d'une vaste expansion formée par la cuticule du parasite.

Les œufs à coque mince $(87 \mu$ sur $48 \mu)$ sont, dans le tissu pulmonaire, à des stades variables d'évolution. Quant aux larves, elles sont enroulées sur elles-mêmes. L'extrémité céphalique est dépourvue de toute ornementation. L'extrémité postérieure, pointue, ondulée, en forme de baïonnette, possède, en outre, une petite épine (planche I, fig. 5) caractéristique des genres Pneumostrongylus et Müllerius.

La taille des spicules, les dimensions relatives du «manche» et de la «lame», la forme et la longueur des côtes antéro-latérale et externo-dorsale permettent de différencier Pneumostrongylus cornigerus de Pneumostrongylus calcaratus, espèce très voisine, organisée à peu près de la même façon et ayant les mêmes localisations.

\section{CONCLUSIONS}

Les auteurs signalent l'existence, dans l'est de la R.C.A., de foyers de pneumonie vermineuse, d'une part chez le Damalisque, et, d'autre part, chez l'Hippotrague et le Bubale rouge qui représentent des hôtes nouveaux.

Le parasite en cause est un Protostrongylidae, Pneumostrongylus cornigerus (12), localisé aux bronchioles et aux alvéoles pulmonaires.

C'est la première fois, au nord de l'équateur, que la maladie est observée chez des ruminants sauvages.

Les lésions sont brièvement décrites.

Les auteurs insistent sur la nécessité d'examiner soigneusement le poumon des Antilopes abattues au sud du dixième parallèle.

\section{Remerciements}

Les auteurs tiennent à remercier vivement Mademoiselle Lestra et Monsieur Gastellu de leur précieuse collaboration. 


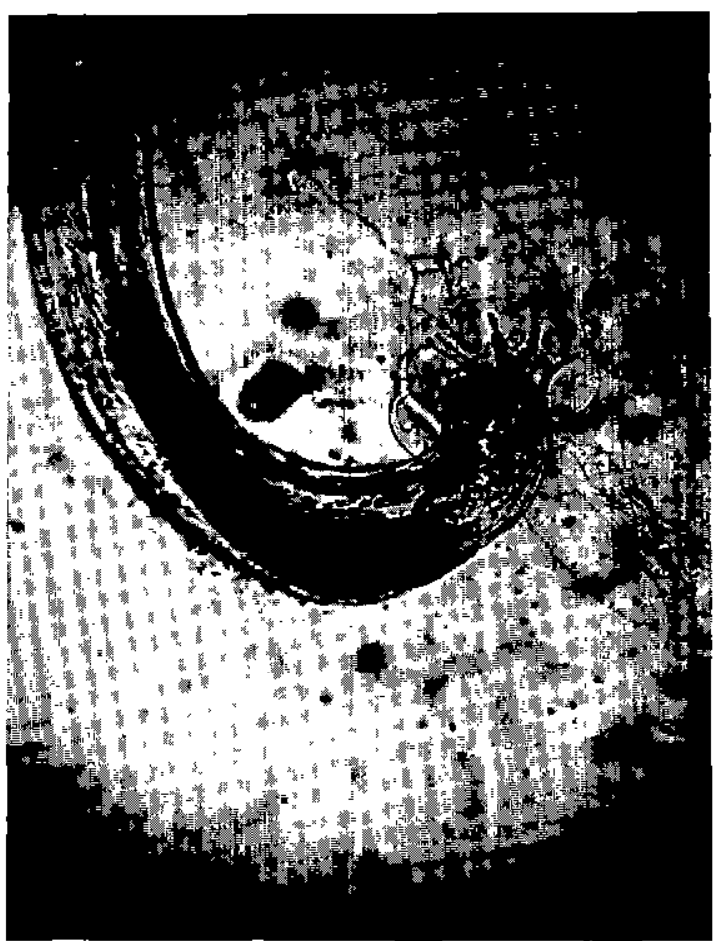

Fig. 1. - Extrémité postérieure du mâle montrant les spicules, le gubernaculum, la bourse caudale et les côtes qui la soutiennent.

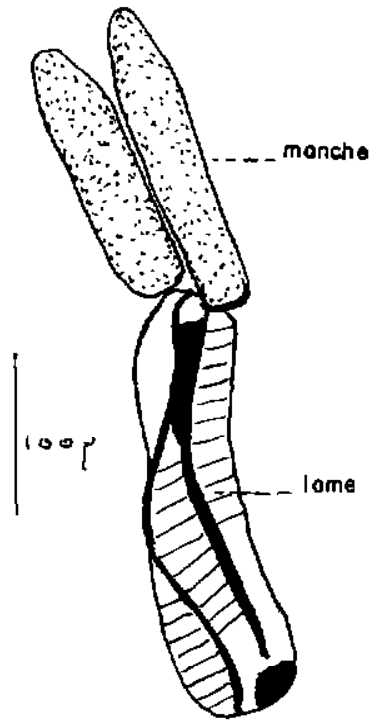

Fig. 3. - Spicules. Détail.

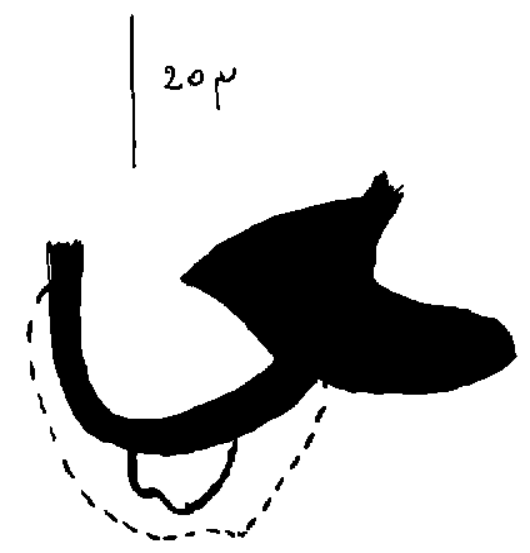

Fig. 2, - Côte dorsale.

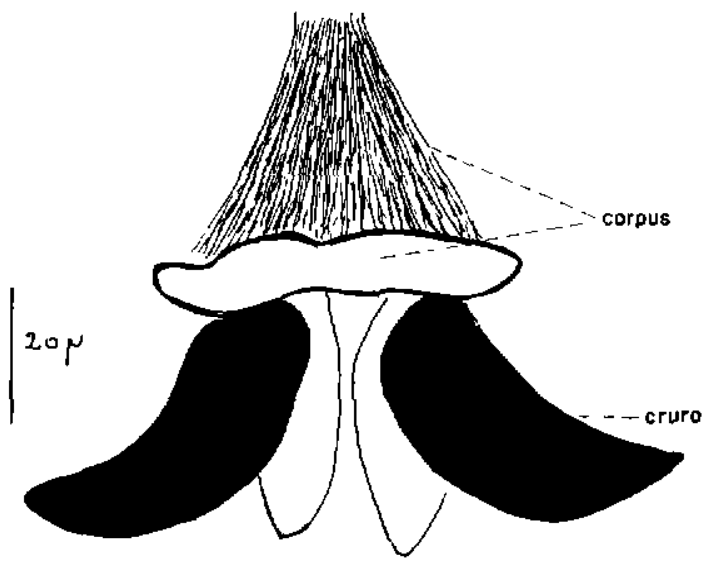

Fig. 4. - Gubernaculum. Détail.

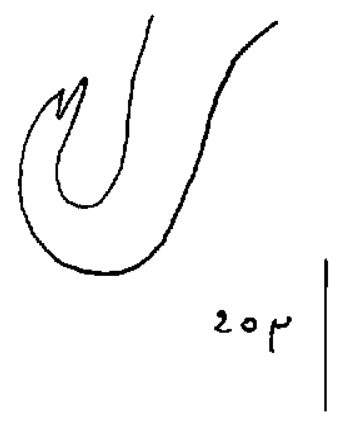

Fig. 5. - Extrémité postérieure d’une larve. 
PL. II. - PNEUMOSTRONGYLUS CORNIGERUS

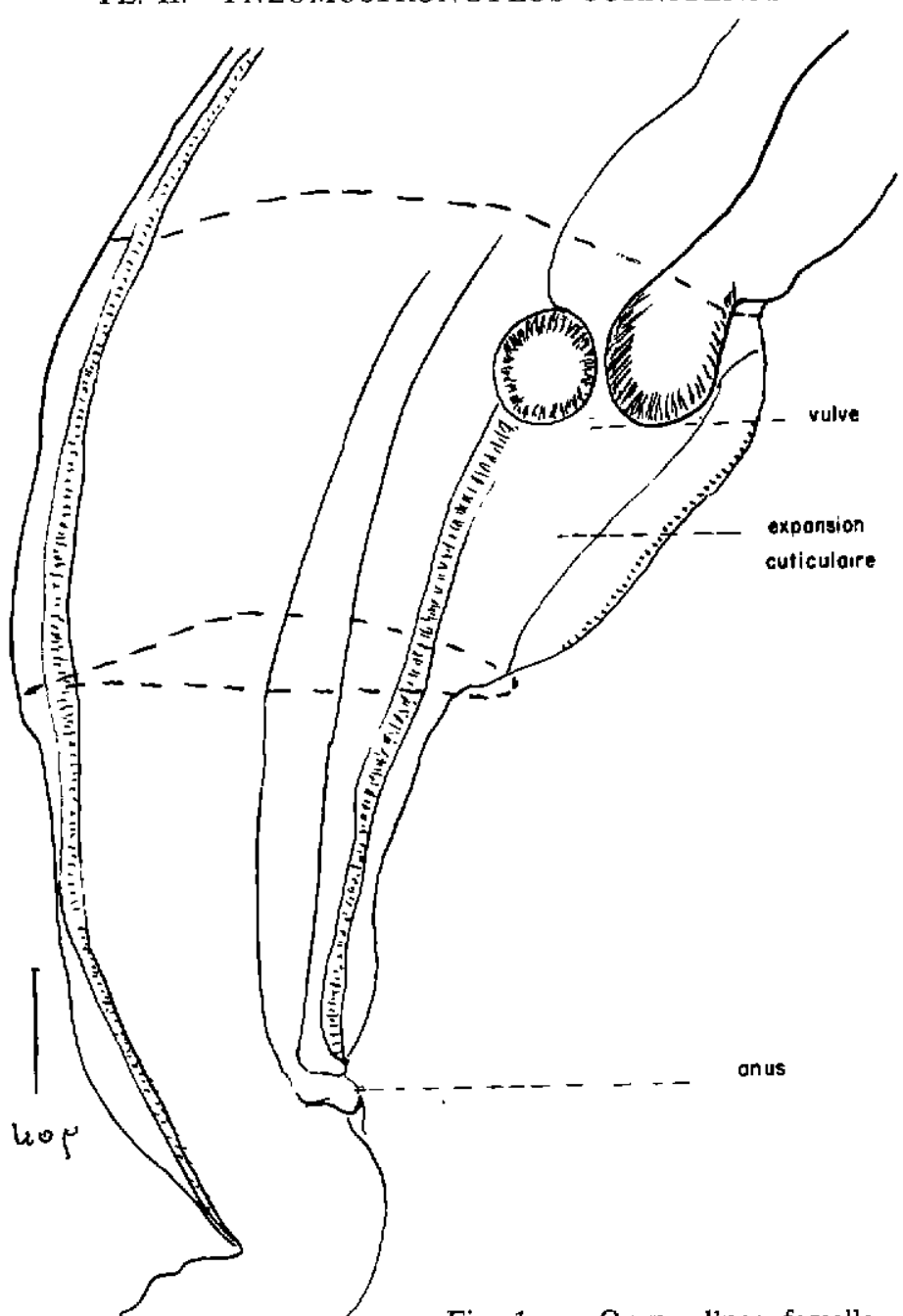

Fig. 1. - Queue d'une femelle.

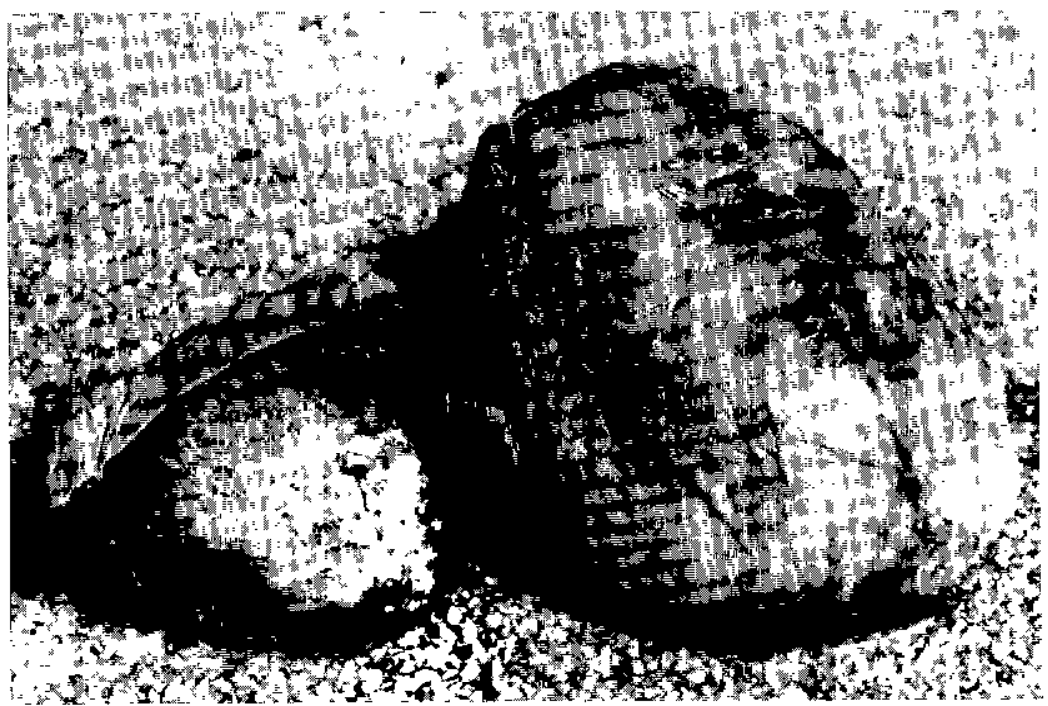

Fig. 2. - Lésions macroscopiques. Nodules isolés. Celui de gauche est sectionné. 


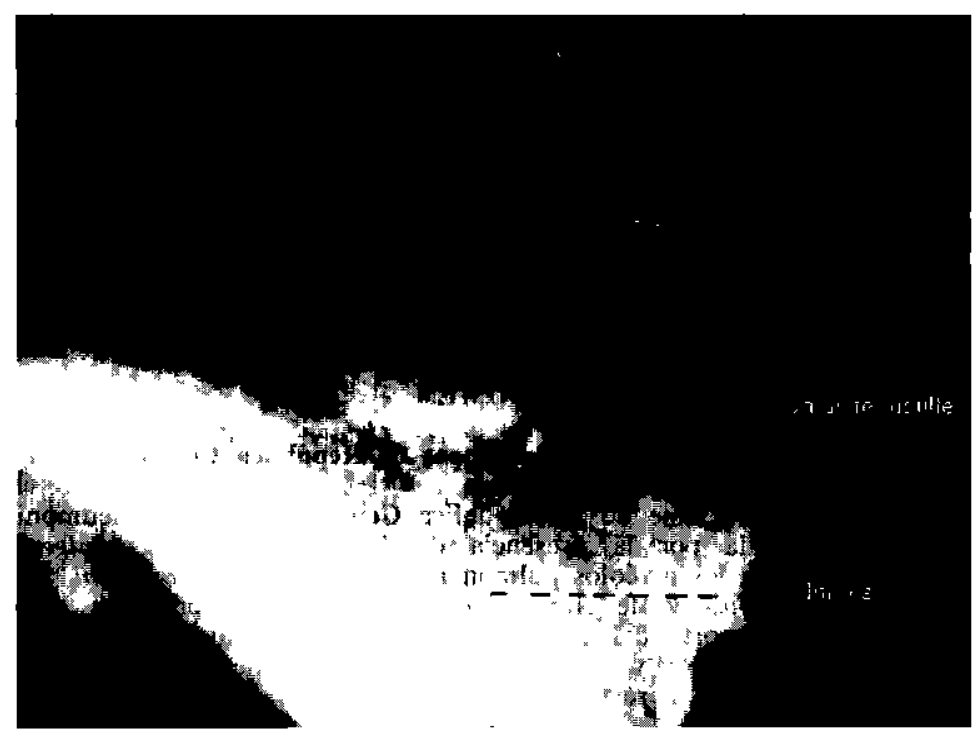

Fig. 1. - Lésion ouverte montrant un parasite adulte et de nombreuses larves.
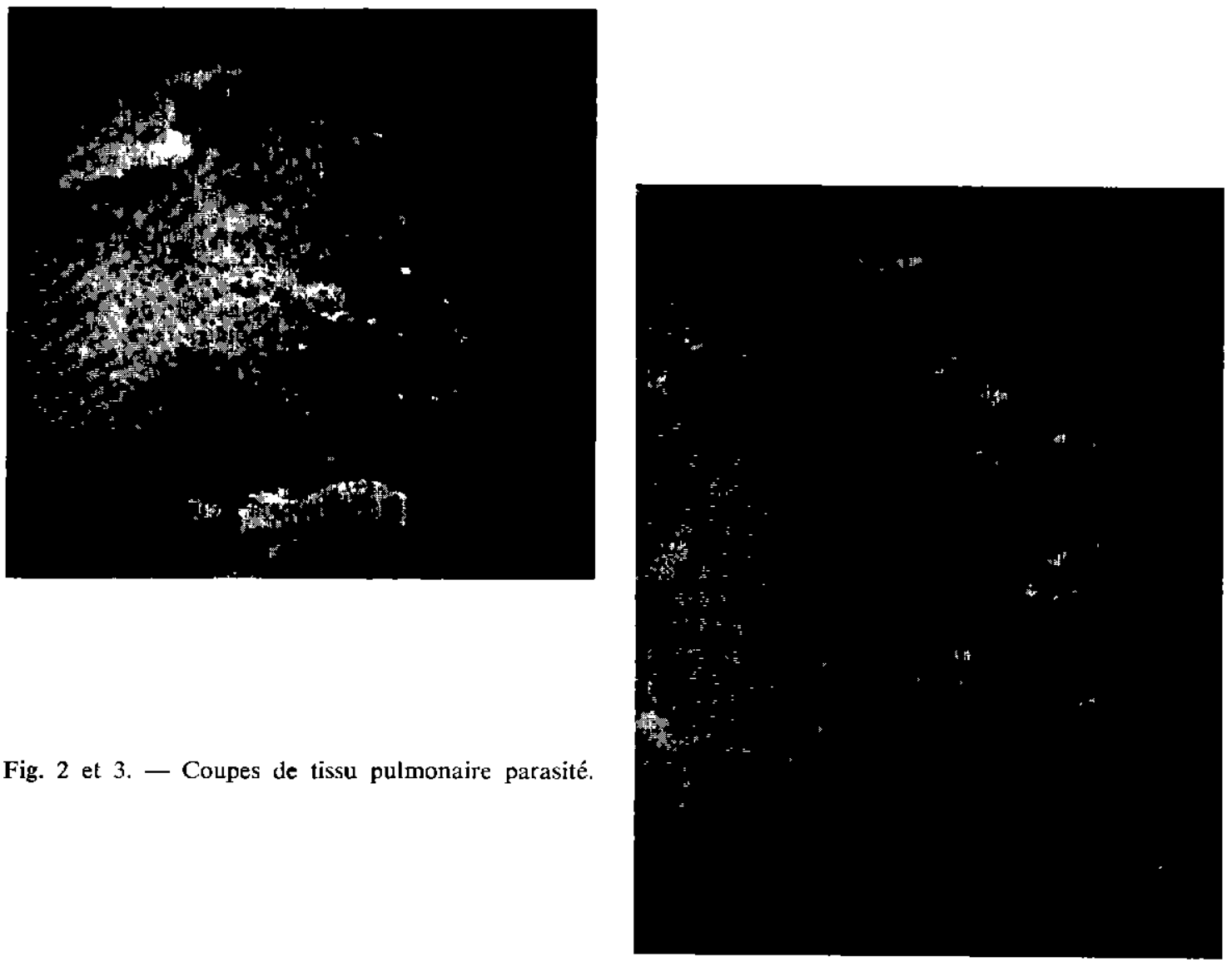

Fig. 2 et 3. - Coupes de tissu pulmonaire parasité. 


\section{SUMMARY}

\section{Lungworms from Central African antelopes}

A verminous pneumonia caused by Pneumostrongylus cornigerus (Ortlepp, 1962) is observed in Antelopes of the subfamilies Alcelaphinae (Damaliscus korrigum and Alcelaphus lelwel) and Oryginae (Hippotragus equinus) shot in the east of the Central african Republic.

Hippotragus equinus and Alcelaphus lelwel are new hosts.

The disease in wild ruminants is mentionned for the first time to the north of the equator.

Parasite and lesions are briefly described.

\section{RESUMEN}

\section{Nemátodos pulmonares de los antílopes de República Centroafricana}

Se señala en el este de la República Centroafricana una neumonia verminosa causada por la presencia de Pneumostrongylus cornigerus (Ortlepp, 1962) en los alvéolos pulmonares de Damaliscus korrigum y de Hippotragus equinus y de Alcelaphus lelwel. Son los primeros casos observados en el norte del ecuador en rumiantes salvajes. Hippotragus equinus y Alcelaphus lelwel representan huespedes nuevos. Se describen de nuevo brevemente parásito y lesiones.

\section{BIBLIOGRAPHIE}

1. DIKMANS (G.). The Springbuck (Antidorcas marsupialis), a new host of the lungworm Bronchonema magna Mönnig, 1932 (Nematoda: Metastrongylidae). Proc. Helminth. Soc. Wash., 1936, 3 (2): 64.

2. DINNIK (J. A.) et SACHS (R.). A gigantic Protostrongylus, Protostrongylus africanus sp. nov. and other lung Nematodes of Antelopes of the Serengeti, Tanzania. Parasitology, 1968, 58 (4) : 819-829.

3. DOUGHERTY (E. C.). A brief survey of the genus Dictyocaulus Railliet et Henry, 1907 ( $\mathrm{Ne}$ matoda: Trichostrongylidae). Proc, Helminth. Soc. Wash., 1946, 13: 49-54.

4. FRECHKOP (S.). Sous-ordre des Ruminants ou Sélénodontes. In: Grassé. Traité de Zoologie, Mammifères. T. 17, Fasc. I, 1955, 568-693.

5. KREIS (H. A.). Die Diagnose des Nematodenbefalles beim Iebenden Rier, mit besonderer Berucksichtigung der Befunde beim Okapi « Bambi ». Acta trop, 1950, 7 (2) : 151-163.

6. MEESER (M.J. N.). A preliminary survey of the endo and ecto parasites of the Impala, Aepyceros melampus. J.S. Afr. vet. med. Ass., 1962, 23 (4) : 221-223.

7. MONNIG (H. O.). Wild Antelopes as carriers of Nematode parasites of domestic ruminants. Part. II. Rep. Dir. Vet. Serv. Anim. Ind. S. Afr. Union, 1932 (a), 1 : 153-171.
8. MONNIG (H. O.). New Strongylid Nematodes of Antelopes. Preliminary notes. J. S. Afr. vet. med. Ass., 1932 (b) : 2 (4) : 171-175.

9. MONNIG (H. O.). Wild Antelopes as carriers of Nematode parasites of domestic ruminants. Onderstepoort J. vet. Res., 1933, 1 (1): 77-92.

10. MOULTON (J. E.) et SACHS (R.). Verminous pneumonia in east african Antelopes. J. comp. Path. Ther., 1970, 80 (1): 169-173.

11. ORTLEPP (R. J.). Encorsig van Suid-afrikaanse helminte weral met verwysing na die wat in ons wilherkouers voorkom. Tydskr. Natuurwet., 1961, 1: $202-212$.

12. ORTLEPP (R. J.). Lungworms from south african Antelopes. Onderstepoort J. vet. Res., 1962, 29 (2) : 173-181.

13. ROUND (M. C.). Check-list of the Helminth parasites of African mammals. Tech. Commun. Commonwealth Bur. Helminth., 1968 (38): 95-97.

14. SACHS (R.) et SACHS (C.). A survey of parasitic infestation of wild herbivores in the Serengeti region in northern Tanzania and the lake Rukwa region in Southern Tanzania, Bull. epizoot. Dis. Afr., 1968, 16 (4) : 455-472.

15. YAMAGUTI (S.). Systema Helminthum. Vol, III, The Nematodes of Vertebrates. Part. I. New York, Inters. Pub. Inc., 1961, pp. 492-3 et 511512.

16. YEH (L. S.). On a collection of Helminths from Thomson's Gazelle, Gazella thomsoni from Tanganyika. J. Helminth., 1956, 29 (4) : 203-228. 